\title{
Mental health of cisgender and transgender during COVID-19 pandemic
}

\author{
Bhattacharjee A. ${ }^{1 *}$, Afshar Jahanshahi A. ${ }^{2}$, Rahman Bhuiyan M. ${ }^{3}$, Sultana.$^{4}$ \\ DOI: https://doi.org/10.17511/ijmrr.2020.i05.02
}

1* Amitab Bhattacharjee, Research Associate, Department of Business Administration, Toyo University, Tokyo, Japan.

2 Asghar Afshar Jahanshahi, Associate Professor, CENTRUM Católica Graduate Business School, Pontificia Universidad Católica del Perú (PUCP), Peru, South America.

3 Mashiur Rahman Bhuiyan, Assistant Register, University of Information Technology and Sciences (UITS), Bangladesh.

4 Sakera Sultana, Resident, Department of Anesthesia, Analgesia and Intensive Care Medicine, Bangabandhu Sheikh Mujib Medical University (BSMMU), Bangladesh.

\begin{abstract}
Normally every individual wants to lead a free life movement but sometimes could not possible due to external environmental effects. During and after the home quarantine, people are still scared by consistent death and infected news of the 2019-nCoV pandemic. Which is why most of the persons have been feeling insecure and nervous that significantly growing up long term stress. The current study depicts the ongoing mental health condition of Bangladeshi cisgender and transgender individuals caused by the SARS-CoV-2 outbreak. Methods: A quarter-long crosssectional survey has been conducted to observe the current mental health condition of the people in Bangladesh. The current study surveyed 1162 participants in the country. The DASS21 scale was used to measure the stress level of individuals. Outcomes: Results show that Bangladeshi individuals experienced a high level of stress (STD-15.28, Mean-32.61) that was higher than other recent studies such as China (STD: 5.45; Mean: 23.65). As a result, high levels of mental stress would be a reason for long-term mental illness after the 2019-nCoV pandemic, which may decline the health and working productivity. However, this study didn't find any significant link between age/education levels and mental stress. Conclusion: The study findings disclosed that the growing up mental stress issue is holding a wide variety of people captive in its claws. Therefore, the study suggests that more studies on mental illness should be required in every region around the world. This may help to make experimental mental health recommendations and possible outlines for starting a healthy life after the COVID-19 pandemic.
\end{abstract}

Keywords: SARS-CoV-2, COVID-19, Mental health, Cisgender and transgender, Bangladesh

Corresponding Author

Amitab Bhattacharjee, Research Associate, Department of Business Administration, Toyo University, Tokyo, Japan.

Email: bhattacharjee.ar@gmail.com
How to Cite this Article

To Browse

Bhattacharjee A, Jahanshahi AA, Bhuiyan MR Sultana S. Mental health of cisgender and transgender during COVID-19 pandemic. Int J Med Res Rev. 2020;8(5):344-351.

Available From

https://ijmrr.medresearch.in/index.php/ijmrr/article/ view/1218

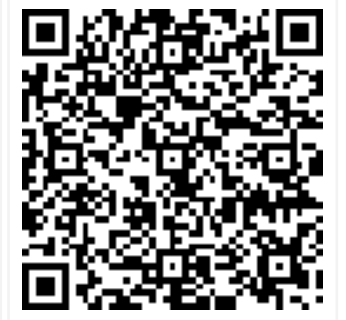

Manuscript Received 2020-09-22

Conflict of Interest No
Review Round 1 2020-09-22

Funding

$\mathrm{Nil}$

Review Round 2
2020-09-30
Ethical Approval
Yes

Yes
Review Round 3

Accepted 2020-10-06

Plagiarism X-checker $5 \%$

(C) 2020 by Amitab Bhattacharjee, Asghar Afshar Jahanshahi, Mashiur Rahman Bhuiyan, Sakera Sultana and Published by Siddharth Health Research and Social Welfare Society. This is an Open Access article licensed under a Creative Commons Attribution 4.0 International License https://creativecommons.org/licenses/by/4.0/ unported [CC BY 4.0] 


\section{Introduction}

At present, our healthy world has been badly suffering from a bio-attack, Coronavirus pandemic $[1,2,3]$. Since February 2020 , one by one all countries except a few have implemented home quarantine policies to protect the people from SARS-CoV-2 infection. Staying at home is enjoyable during holidays but now billions of people are stuck at home to keep themselves safe since the COVID19 pandemic boosts up around the world. Undergoing long-term home quarantine, there is a high possibility to experience mental illness or other health problems $[4,5,6]$. Most recent studies show that cisgender as well as transgender individuals are being stressed due to the SARS-CoV-2 outbreak. People living in developing countries are at high risk of getting extreme levels of stress that might influence their interpersonal sensitivity [2].

Therefore, most of the persons would be more sensitive especially for communicating with others than before. In the literature, some researchers found that cisgender and transgender living in a developing country is mainly stressed due to financial obstacles during the COVID-19 crisis $[7,8]$. The recent mental health research shows that cisgender people are experienced stressed (moderate-severe levels) in China and the high possibility of growing similar mental stress in the other regions $[1,6]$. However, a significant number of cisgender and transgender people had to accept home quarantine due to the Covid-19 pandemic in the developing countries. Many of them have lost jobs or day-to-day working opportunities that spike up financial crises and they become depressed $[2,6,8]$. Currently, the fatality rate caused by 2019 $\mathrm{nCoV}$ in Bangladesh is higher than other badly affected countries like Iran, Italy, France, and the USA.

So, day by day, the situation is getting worse due to a lack of proper medicine and vaccines. Therefore, people are now more scared and stressed by 2019nCoV pandemic news, especially new infected cases and death news in the media. Bangladeshi media confirmed that some people were committed suicide because of mental stress during the COVID-19 lockdown in Bangladesh [2,3]. Furthermore, the media also disclosed that many people living in COVID-19 emergency locations escaped during midnight to other safe areas but unfortunately, a large number of them were infected by 2019-nCoV during this journey [2].
As a result, no area of Bangladesh is now safe or free from SARS-CoV-2. Such negative environmental pressure is significantly increasing the stress level that impacts people's interpersonal sensitivity as well $[2,5]$. Currently, the number of newly infected cases and death caused by Coronavirus has been still increasing in the country. However, during the initial interview session, few participants claimed that in many areas, some people having chronic diseases such as respiratory problems died without clinical care.

Because their family members were forcibly sent them out by assuming that those people were affected by SARS-CoV-2 but not. Adding more, many tenants working at the hospital or clinic or health-related organizations received house rent cancelation notice from their house owners. Especially in the city areas, a significant number of house owners locked their tenants' house/flat and informed them to move out right away whereas some of them put restrictions for health professionals such as doctors, nurses to enter their houses.

During the lockdown, some business/service holders like grocery sellers, medicine retailers, bankers, and representatives of pharmaceutical companies are still working to ensure medicines and food supply in the country but their family members were highly stressed because of the life-safety. Another stressful panic story found that many people were vigorously buying medicines to protect themselves from the COVID-19 attack [2]. This is the current situation of the country due to the high level of stress caused by the COVID-19 pandemic. So the question appears that what would happen if the number of infected cases or death rates or financial crisis keeps on increasing till the following months? Therefore the current study has been conducted to find the ongoing mental illness justification among transgender and cisgender in Bangladesh.

\section{Materials and Methods}

Study method and sampling: The present study followed a cross-sectional survey method (a quarter long) during the 2019-nCoV outbreak in the country in the current calendar. In this study, each participant was randomly selected to collect survey data.

Sample size and survey zone: The current study received complete responses of a total of 1162 participants from all divisions of Bangladesh. 
All the participants from this country are living in the area where the 2019-nCoV was badly spread out and some of them were infected by the Coronavirus.

Survey duration: The current study investigated the depression, anxiety, and stress level of Bangladeshi individuals during ongoing lockdown (from May to July). The total number of infected cases at the beginning of the study was 11,719 and 232,194 was at the end of the study period (Wikipedia).

Survey instrument: Before conducting the survey, the current study used the back translation method to translate the questionnaire including the DASS21 scale from English into Bangla language. Furthermore, the Bangla translated questionnaire by academic experts to measure its reliability was tested.

Then, the Bangla translated version (DASS21 Bangla translated version included in the appendix part) was used for collecting the feedback from all the participants, which was available on the online platform during the survey period.

Data collection techniques: The online survey has been distributed through 'Google Doc', and other social media. Additionally, the postal service was administrated to collect data from transgender people.

Ethical consent: Participation in the study was fully voluntary. The online survey process was anonymously performed and the final online survey included a cover letter/consent form that informed all participants about the main objective of the research. Before conducting the survey, this research was evaluated by the ethics committee of the University of Information Technology and Sciences and then the current study received ethical approval (\#60-211).

Measurements: The present study assessed Depression Anxiety and Stress Scale-21 (DASS21), a mental illness standard survey scale to measure distress, anxiety, and stress, using a range '0-3' [9]. DASS21 comprises three dimensions (Depression Anxiety and Stress) with seven items for each and three different outcome-range: $0-28+$ for distress, 0-20+ for anxiety, and 0-34+ for stress [9].

The participants rated their depression, anxiety, and stress levels by using the DASS21 mental illness scale.
They also filled in their other individual facts such as age, gender, education, having the chronic disease(s), either infected by COVID-19 or not, exercise status, work situation, and financial constraints (developed four items for this study).

Inclusion criteria: Participants experienced or having depression/anxiety/stress were included in this study.

Exclusion criteria: Participants having surgery records within the last six months or less and suffering from other major diseases like blood cancer were excluded from this study.

Statistical tools: The survey data arranged on MS Excel, which was inserted on the STATA (15.0) platform to conduct the study analysis.

\section{Results}

Results in Table 1 indicate the overall stress level of cisgender and transgender living in Bangladesh. In the country, mental stress has significantly increased $(M=32.61)$ due to the 2019-nCoV pandemic, which is higher than China $(M=23.65)$ [1].

Table-1: Regression results by cisgender and transgender.

\begin{tabular}{|c|c|c|c|c|}
\hline \multirow[t]{2}{*}{ Variables } & Fre. & \multicolumn{3}{|c|}{ Regression parameters } \\
\hline & Count(\%) & $\mid \begin{array}{l}\operatorname{Dep}(\beta, \mathrm{LCI} \\
\text { UCI, P) }\end{array}$ & $\begin{array}{l}\operatorname{Anx}(\beta, \mathrm{LCI} \\
\mathrm{UCI}, \mathrm{P})\end{array}$ & $\begin{array}{l}\operatorname{Str}(\beta, \text { LCI, } \\
\text { UCI, P) }\end{array}$ \\
\hline \multicolumn{2}{|c|}{$\begin{array}{l}\text { Depression, Anxiety and Stress } \\
\text { Scale-21 (DASS21) }\end{array}$} & \multicolumn{3}{|c|}{$M=32.61$} \\
\hline Normal level (0-14) & $684(59 \%)$ & \multirow{3}{*}{\multicolumn{3}{|c|}{$\mathrm{n}=1162 \mathrm{STD}=15.28$}} \\
\hline $\begin{array}{l}\text { Mild-moderate level (15- } \\
\text { 25) }\end{array}$ & $221(19 \%)$ & & & \\
\hline $\begin{array}{l}\text { Severe-extremely severe } \\
\text { level }(26-34+)\end{array}$ & $257(22 \%)$ & & & \\
\hline \multicolumn{5}{|l|}{ Gender } \\
\hline Transgender & $62(5 \%)$ & \multicolumn{3}{|c|}{ Reference group } \\
\hline $\begin{array}{l}\text { Cisgender } \\
\text {-Female }\end{array}$ & $379(33 \%)$ & $\begin{array}{l}-2.61 \\
1.81\end{array}$ & $\begin{array}{l}-3.60 \\
2.88\end{array}$ & $\begin{array}{l}-5.50 \\
-3.74\end{array}$ \\
\hline $\begin{array}{l}\text { Cisgender } \\
\text {-Male }\end{array}$ & $721(62 \%)$ & $\begin{array}{l}-2.40 \\
0.079\end{array}$ & $\begin{array}{l}-3.35 \\
0.153\end{array}$ & $\begin{array}{l}-7.26 \\
0.000\end{array}$ \\
\hline
\end{tabular}

Note: $* \mathrm{p}<0.05, * * \mathrm{p}<0.01, * * * \mathrm{p}<0.001$

Dep: Depression; Anx: Anxiety; Str: Stress; Fre: Frequency

The depression ( 7 items), anxiety ( 7 items), and stress ( 7 items) of DASS21 had Cronbach's alpha value of $.971, .960$, and .973 respectively. 
According to the statistical outcomes, approximately $62(5 \%)$ transgender people responded to our survey were highly stressed compared to cisgenderfemale $(n=379 ; 33 \%)$ and cisgender-male $(n=721$; $62 \%$ ) individuals during the COVID-19 pandemic in the country. Following the cut-off scores of stress level in DASS21, 684 (59\%) and 257 (22\%) of the participants had normal levels and severe to extremely severe mental stress respectively. As seen in Table 1, it was found that cisgender participants had a lower level of stress in comparison to transgender individuals $(\beta=-5.50$, $p=0.000,95 \% C I-3.74$ to -7.26$)$.

Table-2: OLS Regression results by age and education.

\begin{tabular}{|c|c|c|c|c|}
\hline \multirow[t]{2}{*}{ Variables } & \multirow{2}{*}{\begin{tabular}{|c|} 
Fre. \\
Count(\%)
\end{tabular}} & \multicolumn{3}{|c|}{ Regression parameters } \\
\hline & & $\begin{array}{l}\text { Dep ( } \beta, \text { LCI, } \\
\text { UCI, P) }\end{array}$ & $\begin{array}{l}\text { Anx ( } \beta, \text { LCI, } \\
\text { UCI, P) }\end{array}$ & $\begin{array}{l}\operatorname{Str}(\beta, \text { LCI, } \\
\text { UCI, P) }\end{array}$ \\
\hline \multicolumn{2}{|l|}{ Age } & \multicolumn{3}{|c|}{$n=1162$} \\
\hline $21-30$ years old & $519(45 \%)$ & \multirow{5}{*}{$\begin{array}{c}0.36 \\
0.48 \\
-0.63 \\
1.359\end{array}$} & \multirow{5}{*}{$\begin{array}{c}0.32 \\
0.65 \\
-1.29 \\
0.518\end{array}$} & \multirow{5}{*}{$\begin{array}{c}-0.56 \\
0.43 \\
-1.54 \\
0.267\end{array}$} \\
\hline $31-40$ years old & $384(33 \%)$ & & & \\
\hline $41-50$ years old & $211(18 \%)$ & & & \\
\hline $51-60$ years old & $39(3 \%)$ & & & \\
\hline Over 60 years old & $9(1 \%)$ & & & \\
\hline \multicolumn{2}{|l|}{ Education level } & \multicolumn{3}{|c|}{$n=1162$} \\
\hline \multicolumn{2}{|c|}{\begin{tabular}{|l|l|}
$\begin{array}{l}\text { Primary level/School } \\
\text { dropout* }\end{array}$ & \\
\end{tabular}} & \multirow{6}{*}{$\begin{array}{c}-0.18, \\
0.86 \\
-1.50 \\
0.394\end{array}$} & \multirow{6}{*}{$\begin{array}{c}0.30 \\
0.95 \\
-1.37 \\
0.381\end{array}$} & \multirow{6}{*}{$\begin{array}{c}-0.50 \\
0.17 \\
-1.16 \\
0.143\end{array}$} \\
\hline $\begin{array}{l}\text { Higher secondary } \\
\text { level }\end{array}$ & $148(12.7 \%)$ & & & \\
\hline $\begin{array}{l}\text { 2-year college } \\
\text { degree/ diploma }\end{array}$ & $135(11.6 \%)$ & & & \\
\hline Bachelor degree & $486(41.8 \%)$ & & & \\
\hline Master degree & $317(27.3 \%)$ & & & \\
\hline $\mathrm{PhD}$ & $5(0.4 \%)$ & & & \\
\hline
\end{tabular}

Note: $* p<0.05, * * p<0.01, * * * p<0.001$

Dep: Depression; Anx: Anxiety; Str: Stress; Fre: Frequency

School dropout: An individual who attended elementary school but left education without obtaining a basic credential level (in/after a primary level or in/before higher secondary level).

Considered the current study outcomes (Table 2), the age and education levels of the participants had no direct impact on changing stress level (age: $\beta=$ -0.56, $p=0.267,95 \%$ CI 0.43 to -1.54 ; education: $\beta=-0.50, p=0.143,95 \%$ CI 0.17 to -1.16$)$. As seen in the Statistical distribution in Table 3, it was also found that the participants $(54 \% ; n=628)$ were unsure, whether they were infected by '2019-nCoV' or not.
$31 \%(n=356)$ of the participants responded as 'NO' infected status.

Table-3: OLS Regression results by COVID infected and Chronic disease.

\begin{tabular}{|c|c|c|c|c|}
\hline \multirow[t]{2}{*}{ Variables } & \multirow{2}{*}{\begin{tabular}{|c|} 
Fre. \\
Count (\%)
\end{tabular}} & \multicolumn{3}{|c|}{ Regression parameters } \\
\hline & & $\begin{array}{l}\operatorname{Dep}(\beta, \text { LCI, } \\
\text { UCI, P) }\end{array}$ & $\begin{array}{l}\text { Anx }(\beta, \text { LCI, } \\
\text { UCI, P) }\end{array}$ & $\begin{array}{l}\text { Str }(\beta, \text { LCI, } \\
\text { UCI, P) }\end{array}$ \\
\hline \multicolumn{2}{|c|}{ Infected by COVID-19 } & \multicolumn{3}{|c|}{$\mathrm{n}=1162$} \\
\hline No & $356(31 \%)$ & $\begin{array}{l}2.98 \\
1.01\end{array}$ & $\begin{array}{l}2.77 \\
1.42\end{array}$ & $\begin{array}{l}\text { 5.59, } \\
4.01,\end{array}$ \\
\hline Yes & $178(15 \%)$ & $\begin{array}{l}3.11 \\
0.010\end{array}$ & $\begin{array}{l}-3.51, \\
0.094\end{array}$ & $\begin{array}{l}7.17, \\
0.000\end{array}$ \\
\hline Unsure & $628(54 \%)$ & \multicolumn{3}{|c|}{ Reference group } \\
\hline \multicolumn{2}{|c|}{$\begin{array}{l}\text { Current-chronic disease } \\
\text { status }\end{array}$} & \multicolumn{3}{|c|}{$n=1162$} \\
\hline Type 1 & $519(45 \%)$ & \multicolumn{3}{|c|}{ Reference group } \\
\hline Type 2 & $134(12 \%)$ & $\begin{array}{l}2.36 \\
0.84 \\
4.09 \\
0.006\end{array}$ & $\begin{array}{c}2.54 \\
0.48 \\
-4.60 \\
0.116\end{array}$ & $\begin{array}{c}2.15 \\
0.72 \\
-2.59 \\
0.087\end{array}$ \\
\hline Type 3 & $394(34 \%)$ & $\begin{array}{c}2.07 \\
0.23 \\
-3.26 \\
0.127\end{array}$ & $\begin{array}{c}1.14 \\
0.08 \\
-2.31 \\
0.229\end{array}$ & $\begin{array}{l}2.99, \\
0.70 \\
4.88 \\
0.002\end{array}$ \\
\hline Type 4 & $115(10 \%)$ & $\begin{array}{c}1.95 \\
0.58 \\
-2.14 \\
0.096\end{array}$ & $\begin{array}{c}1.21 \\
0.31 \\
-1.42 \\
0.237\end{array}$ & $\begin{array}{l}3.78 \\
0.92 \\
5.33 \\
0.000\end{array}$ \\
\hline
\end{tabular}

Note: $* \mathrm{p}<0.05, * * \mathrm{p}<0.01, * * * \mathrm{p}<0.001$

Dep: Depression; Anx: Anxiety; Str: Stress; Fre: Frequency

Type 1: Do not have any chronic disease.

Type 2: Frequent colds/seasonal flu and digestive problems,

Type 3: Ordinary/common blood-related problems such as Blood sugar/Diabetes.

Type 4: Loss of sexual desire, body pain, or disorder related problems such as migraine, headaches, chest pain and rapid heartbeat, Insomnia/sleeping disorder.

The statistical evaluation shows that the participants with unsure status had a higher stress level than the participants with negative status (Depression: $\beta=2.98, p=0.010,95 \%$ CI 1.01 to 3.11; Stress: $\beta=5.59, p=0.000,95 \%$ CI 4.01 to 7.17$)$. In this study, around $15 \%(n=178)$ of the participants ensured that they were positive for the SARS-CoV-2 virus/COVID-19. 
By the current-chronic disease status, $55.30 \%$ ( $n=643$ ) of the participants had the chronic disease (rounded up including type 2, 3, and 4) while the rest of them $(44.70 \% ; n=519)$ were reported as the negative status of chronic disease.

Furthermore, results were depicted that the participants with chronic disease had more stress than healthy individuals (Type 2: $\beta=2.36, p=0.006$, 95\% CI 0.84 to 4.09 ; Type 3: $\beta=2.99, p=0.002$, $95 \%$ CI 0.70 to 4.88 ; Type $4: \beta=3.78, p=0.000$, $95 \%$ CI 0.92 to 5.33 ), which was also found positive in the previous study [6].

By daily exercise status (Table 4), Bangladeshi cisgender and transgender individuals who exercised one or more hours regularly stated that they had mostly normal mental condition than those who exercised zero-hour daily $(\beta=-3.46, p=0.000$, $95 \% C I-2.04$ to -4.98$)$. In this study, it was found that around $59 \%(n=687)$ of the participants were confirmed with no exercise status.

Table-4: OLS Regression results by exercise and employment status.

\begin{tabular}{|c|c|c|c|c|}
\hline \multirow[t]{2}{*}{ Variables } & Fre. & \multicolumn{3}{|c|}{ Regression parameters } \\
\hline & Count (\%) & $\begin{array}{l}\text { Dep ( } \beta \text {, } \\
\text { LCI, UCI, } \\
\text { P) }\end{array}$ & $\begin{array}{l}\text { Anx ( } \beta \text {, } \\
\text { LCI, UCI, } \\
\text { P) }\end{array}$ & $\begin{array}{l}\text { Str ( } \beta, \\
\text { LCI, UCI, } \\
\text { P) }\end{array}$ \\
\hline \multicolumn{2}{|l|}{ Daily exercise hours } & \multicolumn{3}{|c|}{$n=1162$} \\
\hline 0 Hour & $687(59 \%)$ & \multicolumn{3}{|c|}{ Reference group } \\
\hline 1 Hour & $339(29 \%)$ & \multirow{4}{*}{$\begin{array}{l}-3.54 \\
2.10 \\
-3.98 \\
0.089\end{array}$} & \multirow{4}{*}{$\begin{array}{l}-3.37 \\
1.97 \\
-2.76 \\
0.062\end{array}$} & \multirow{4}{*}{$\begin{array}{l}-3.46 \\
-2.04 \\
-4.98 \\
0.000\end{array}$} \\
\hline 2 Hours & $105(9 \%)$ & & & \\
\hline 3 Hours & $19(2 \%)$ & & & \\
\hline More than 3 Hours & $12(1 \%)$ & & & \\
\hline \multicolumn{2}{|l|}{ Work situation } & \multicolumn{3}{|c|}{$n=1162$} \\
\hline $\begin{array}{l}\text { Unemployed or stopped or } \\
\text { lost the job due to COVID-19 }\end{array}$ & $597(51 \%)$ & \multicolumn{3}{|c|}{ Reference group } \\
\hline Working at office & $362(31 \%)$ & $\begin{array}{l}-2.03 \\
1.66\end{array}$ & $\begin{array}{l}-1.91 \\
1.87\end{array}$ & $\begin{array}{l}-2.93 \\
-1.81\end{array}$ \\
\hline Working from home & $203(17 \%)$ & $\begin{array}{l}-2.01 \\
0.059\end{array}$ & $\begin{array}{l}-2.62 \\
0.219\end{array}$ & $\begin{array}{l}-3.09 \\
0.000\end{array}$ \\
\hline
\end{tabular}

Note: $*_{p}<0.05, * * p<0.01, * * * p<0.001$

Dep: Depression; Anx: Anxiety; Str: Stress; Fre: Frequency

By the working situation (Table 4), most of the participants (51\%; $n=597) \quad$ had stopped/lost/unemployed working status due to the COVID-19 pandemic. They experienced high levels of stress compared to individuals working at office $(31 \% ; n=362)$ and from home $(17 \% ; n=203)(\beta=$ $-2.93, p=0.000,95 \%$ CI -1.81 to -3.09 ).
Table-5: Regression results from financial constraints and mental illness

\begin{tabular}{|c|c|c|c|c|}
\hline \multirow[t]{2}{*}{ Variables } & \multirow[t]{2}{*}{ Fre. } & \multicolumn{3}{|c|}{$\begin{array}{l}\text { Regression } \\
\text { parameters }\end{array}$} \\
\hline & & $\begin{array}{l}\operatorname{Dep}(\beta, \\
\text { LCI, } \\
\text { UCI, P) }\end{array}$ & $\begin{array}{l}\text { Anx }(\beta, \\
\text { LCI, } \\
\text { UCI, P })\end{array}$ & $\begin{array}{l}\operatorname{Str}(\beta, \\
\text { LCI, } \\
\text { UCI, P) }\end{array}$ \\
\hline \multicolumn{5}{|l|}{ Financial constraints } \\
\hline $\begin{array}{l}\text { I do not have sufficient income to } \\
\text { meet the basic needs in my life }\end{array}$ & $n=1162$ & \multicolumn{3}{|c|}{ Reference group } \\
\hline $\begin{array}{l}\text { My earnings is insufficient to cover } \\
\text { mine monthly expenses }\end{array}$ & $n=1162$ & \multirow{3}{*}{$\begin{array}{l}-3.91 \\
-1.03 \\
-4.96 \\
0.000\end{array}$} & \multirow{3}{*}{$\begin{array}{l}-1.77 \\
0.19 \\
-1.62 \\
0.109\end{array}$} & \multirow{3}{*}{$\begin{array}{l}-4.81 \\
-2.02 \\
-5.99 \\
0.000\end{array}$} \\
\hline $\begin{array}{l}\text { I have unpaid borrowings that are } \\
\text { used for basic items such as food, } \\
\text { living expenses, etc. }\end{array}$ & $n=1162$ & & & \\
\hline $\begin{array}{l}\text { My earnings are sufficient to lead my } \\
\text { life }\end{array}$ & $n=1162$ & & & \\
\hline
\end{tabular}

Note: $* p<0.05, * * p<0.01, * * * p<0.001$

Dep: Depression; Anx: Anxiety; Str: Stress; Fre: Frequency

By the predictors of financial constraints, Bangladeshi cisgender and transgender individuals with financial or daily income-related problems such as do not have sufficient income to meet the basic needs had a high level of mental stress than cisgender or those who had sufficient income $(\beta=$ -4.81, $p=0.000,95 \% \quad C I-2.02$ to -5.99 ). Specifically, transgender people were in a dire situation because of less access to daily income, which has been statistically evidenced in Table 5 .

\section{Discussion}

In the current COVID-19 pandemic, the research on mental illness of cisgender and transgender individuals is very insufficient $[2,6,7,10]$. A similar study would be effective to know during and after the 2019-nCoV pandemic $[6,7,10]$. Moreover, health, life satisfaction, negative or positive thinking, and interpersonal sensitivity might be significantly changed by severe levels of mental stress $[5,6]$. Therefore, the study findings provide current mental health knowledge in a similar context. This study denotes that Bangladeshi cisgender and transgender (DASS21: SD-15.28, Mean-32.61) were more stressful during the lockdown because of the COVID-19 pandemic in Bangladesh. In the study sample, severe-extremely severe mental stress and mild-moderate mental stress were found around $22 \%$ and $19 \%$ respectively, whereas approximately $59 \%$ of participants were normal mental health. 
One of the studies ' relevant facts is that age and education levels haven't any significant impact on mental stress during an external or uncontrollable environmental pandemic but the other major differences in stress levels are denoted and significant by infected status, financial constraints, and exercise. However, the social changes such as long term restrictions on moving out and limited access to outside dramatically have reduced the income or day-to-day working opportunity mostly for transgender people than cisgender individuals in the country.

Therefore, the home quarantine procedure was like a prison life for all. This rising issue had vastly developed mental illness among cisgender as well as transgender people and reasonably they experienced high levels of stress. According to the results distributed in Table 2, similar to previous research, the current study found that mental illness might not be developed surprisingly because of any change in age or education level $[1,6]$. In contrast, doing daily exercise less or more significantly reduces mental stress (Table 4), which is found relevant to other recent mental health studies $[1,5,6]$.

The study results also showed that individuals who stopped working during the lockdown were more stressed than individuals working from home (online desk) or having other working sources for income. This evidence is also supported by other research statistics in the literature [6]. In the literature, many scholars showed that having less income or working opportunities increases mental distress.

However, the current or long-term chronic disease is significantly correlated with the increasing stress level among cisgender and transgender individuals whether male or female. For example, long term sleep disturbance or insomnia reduces work abilities and mental fitness [11].

The regression distribution of the study shows that participants with chronic disease such as Loss of sexual desire, body pain or disorder related problems such as migraine, headaches, chest pain, and rapid heartbeat, Insomnia/sleeping disorder had experienced a high level of stress than attendants having common chronic diseases like Blood sugar/Diabetes, frequent colds/seasonal flu. Other recent investigations also mentioned the same positive correlation between chronic disease and mental stress among people [6].
As next, by COVID-19 infected, participants in the study with no infected status were found normal mental health conditions but found moderate-severe levels of stress among transgender and cisgender who were positive with SARS-CoV-2 virus. Finally, the statistical findings in Table 5 show that cisgender and transgender individuals having financial problems are most stressed, whereas individuals having near to zero or minimum financial problems are likely distressed.

In the literature, the research on stress and hormonal change context suggest that experiencing long term stress (specifically two or more than two weeks) would reduce the working ability [11]. So, regaining normal mental health, an individual may need almost a month but some cases would require nearly six months to a year (in serious condition) $[4,11]$.

Therefore, the study suggests that the working/living condition and working/regular functioning pressure of an individual should be flexible during the first working month after the COVID-19 outbreak. Thus, everyone would regain better mental health after the pandemic.

\section{Limitations}

Similar to other research studies, this study faced some limitations. Firstly, the present study was derived from evidence on selected divisions by a cross-sectional survey. Therefore, the study findings are predictable only. Secondly, in the present study sample, several participants were found as infected by SARS-CoV-2 but the number of those participants wasn't really large. Thirdly, the study results show a comprehensive overview of severe levels (focus on stress) of mental illness caused by the COVID-19 pandemic but some of the participants already had similar stress problems due to their previous chronic disease or economic constraints.

\section{Conclusion}

To sum up, the current study findings disclose that the mental illness caused by the COVID-19 outbreak is significantly higher among Bangladeshi transgender than cisgender but experiencing depression or stress might disrupt the regular activities of both groups. Furthermore, both cisgender and transgender individuals are mainly being stressed because of financial constraints rather than COVID-19 fear. 
Therefore, high levels of stress would negatively influence the health and work efficiency as well as the regular life activities of many individuals during or after the COVID-19 pandemic. So, this study recommends adopting daily exercise and positive thinking techniques to reduce mental stress and start a healthy life again. Lastly, the current research also suggests that transgender individuals may need more financial support than cisgender people to reduce their long term mental distress, which would truly increase their life satisfaction.

\section{What does the study add to the existing knowledge}

In the literature, this study would help to increase knowledge on the mental stress condition of cisgender and transgender due to virus fear and financial constraints in the developing country during the COVID-19 pandemic.

\section{Author's contribution}

Amitab Bhattacharjee: Conceptualization, Investigation, Resources, Methodology, Formal Analysis, Visualization, Writing- Original, Review, and Editing.

Asghar Afshar Jahanshahi: Visualization, WritingReview, and Editing.

Mashiur Rahman Bhuiyan: Investigation (data collection and developing database)

Sakera Sultana: Investigation (data collection), Writing-Review, and Editing.

\section{Acknowledgment}

The authors are grateful to the medical staff at BSMMU Hospital and Dhaka medical college and hospital (DMCH). The authors are also thankful to Professor Dr. A. N. M. Shareef, at the University of Information Technology and Sciences (UITS), Rupa Bhattacharjee at National University, and Dr. Satabdi Datta at Square Hospital, in Bangladesh.

\section{Reference}

01. Bao Y, Sun Y, Meng S, Shi J, Lu L. 2019-nCoV epidemic address mental health care to empower society. Lancet. 2020;395(10224)e37e38.

doi: $10.1016 / S 0140-6736(20) 30309-3$ [Crossref]
02. Mamun MA, Griffiths MD. First COVID-19 suicide case in Bangladesh due to fear of COVID-19 and xenophobia- Possible suicide prevention strategies. Asian J Psychiatr. 2020;51;102073. doi: 10.1016/j.ajp.2020.102073 [Crossref]

03. Somoy News. Youth Suicide in Gaibandha Due to Doubt of Corona (In Bangla). Retrieved on March 31, 2020.

Available from: [Article] [Crossref]

04. Ruiz FJ, Martín MBG, Falcón JCS, González PO. The hierarchical factor structure of the Spanish version of Depression Anxiety and Stress Scale21. Int J Psychol Ther. 2017;17(1)97-105. Retrieved from [Article] [Crossref]

05. Xiang YT, Yang Y, Li W, Zhang L, Zhang Q, Cheung $\mathrm{T}, \mathrm{Ng} \mathrm{CH}$. Timely mental health care for the 2019 novel coronavirus outbreak is urgently needed. Lancet Psychiatry. 2020;7(3)228-229.

doi: $10.1016 / S 2215-0366(20) 30046-8$ [Crossref]

06. Zhang SX, Wang $Y$, Rauch A, Wei F. Unprecedented disruption of lives and workHealth, distress and life satisfaction of working adults in China one month into the COVID-19 outbreak. Psychiatry Res. 2020;288;112958.

doi: $10.1016 /$ j.psychres.2020.112958 [Crossref]

07. Stenqvist T. The social struggle of being HIJRA in Bangladesh-cultural aspiration between inclusion and illegitimacy. 2015.

Retrieved from: [Article] [Crossref]

08. Dhakatribune. Coronavirus- Transgenders hit hard as lockdown bites. 2020.

Retrieved from: [Article] [Crossref]

09. Antony MM, Bieling PJ, Cox BJ, Enns MW and Swinson RP. Psychometric properties of the 42item and 21-item versions of the Depression Anxiety Stress Scales in clinical groups and a community sample. Psychol Assess. $1998 ; 10(2) 176$.

doi: $\quad 10.1037 / 1040-3590.10 .2 .176 \quad$ [Crossref] 
10. Khan SI, Hussain MI, Parveen S, Bhuiyan MI, Gourab G, Sarker GF, Arafat SM, Sikder J. Living on the extreme margin social exclusion of the transgender population (hijra) in Bangladesh. J Health Popul Nutr. 2009;27(4)441.

[Crossref]

11. Fortunato VJ, Harsh J. Stress and sleep qualityThe moderating role of negative affectivity. Personality and Individual Differences. 2006;41(5)825-836.

[Crossref]

\section{Appendix}

Table A: The Depression Anxiety and Stress Scale-21(DASS21) in English.

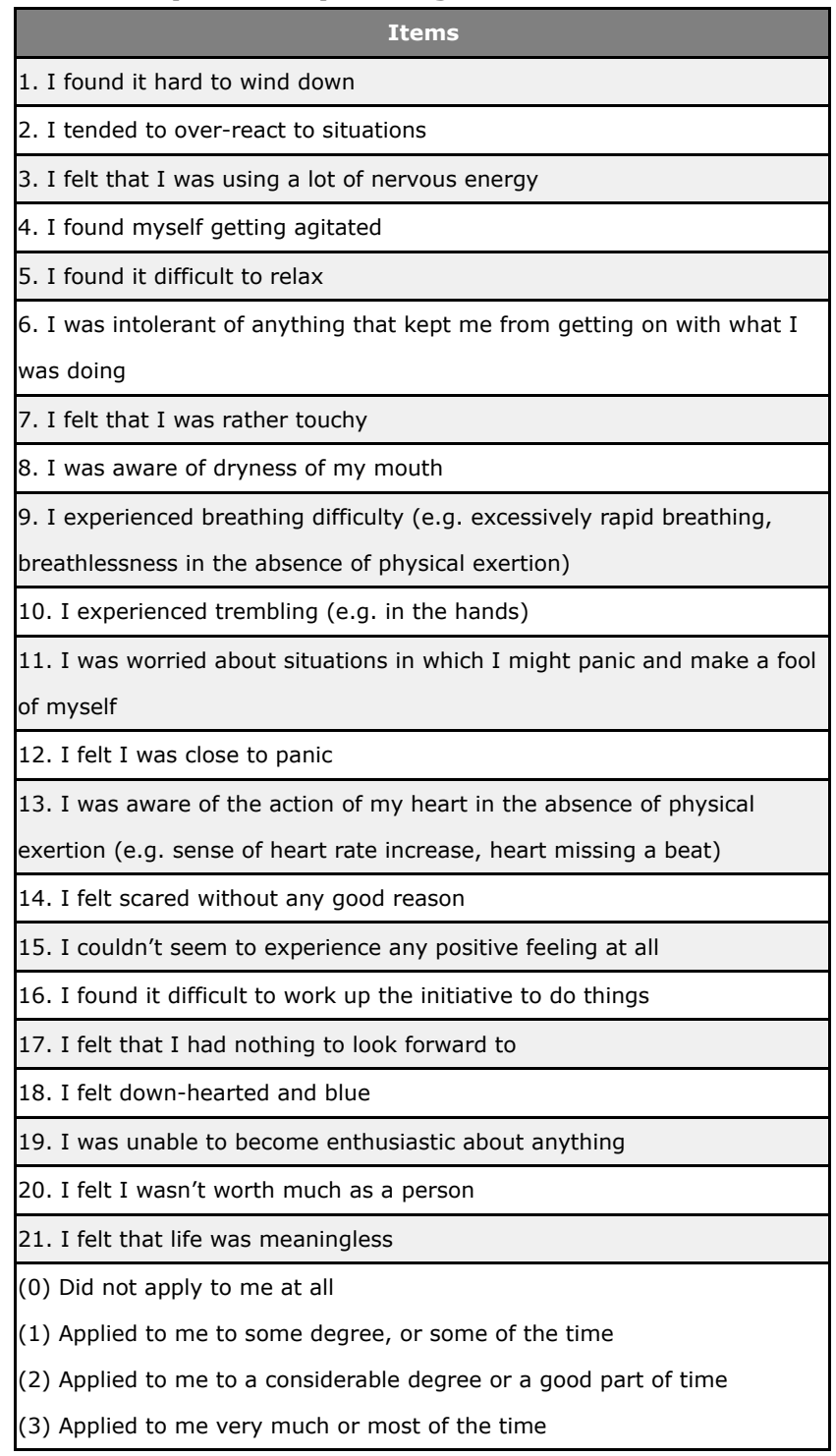

Table B: The Depression Anxiety and Stress Scale-21(DASS21) in Bangla.

\begin{tabular}{|c|c|}
\hline Items & Consistency by a \\
\hline ১. আমার নিজেকে শান্ত রাখতে কষ্ট হয়েছে। & \multirow[t]{2}{*}{$a>0.90$} \\
\hline ২. আমি বিভিন্ন সময়ে অতিমাত্রায় সংবেদনশীল ছিলাম। & \\
\hline
\end{tabular}

\begin{tabular}{|c|c|}
\hline ৩. আমার মনে হতো আমি অনেক বেশি স্নায়ুবিক চাপে আছি । & \\
\hline 8. আমি অল্পতেই উত্তেজিত হয়ে পরতাম। & \\
\hline ৫. আমি নিজেকে সহজে শান্ত করতে পারতাম না । & \\
\hline ৬. আমি সহ্য করতে পারতাম না যে, যা করতে চাইতাম তা থেকে আমাকে & \\
\hline কিছু বিরত রাখত । & \\
\hline ৭. আমার মনে হতো যে, আমি স্পর্শকাতর । & \\
\hline ৮. আমার গলা শুকিয়ে আসত । & \multirow{7}{*}{$a>0.90$} \\
\hline $\begin{array}{l}\text { ৯. আমার শ্বাসকষ্ট হতো (অতিদ্রুত শ্বাস-প্রশ্বাস, কাজ না করেও হাঁপিয়ে } \\
\text { উঠা)। }\end{array}$ & \\
\hline ১০. আমার হাত পা কাঁপতো। & \\
\hline $\begin{array}{l}\text { ১১. আমি এমন সব অবস্থা নিয়ে দুশ্চিন্তাগ্রস্থ ছিলাম, যাতে আমি ঘাবড়ে } \\
\text { যেতে পারি বা বোকামি করে ফেলতে পারি । }\end{array}$ & \\
\hline ১২. আমার মনে হতো যে আমি আতঙ্কগ্রস্ত হয়ে যাচ্ছি। & \\
\hline $\begin{array}{l}\text { ১৩. স্বাভাবিক সময়েও (শারীরিক কসরত না করা অবস্থায়) আমি আমার } \\
\text { হৃদ্পন্দণ অনুভব করতে পারতাম (হৃদস্পন্দন বেড়ে যাওয়া, হৃদস্পন্দন } \\
\text { অনিয়মিত হয়ে যাওয়া)। }\end{array}$ & \\
\hline ১৪. আমি অকারণে ভয় পেয়ে যেতাম । & \\
\hline ১৫. আমি কোন কিছুতেই আগ্রহ পেতাম না । & \multirow{7}{*}{$a>0.90$} \\
\hline ১৬. আমি যে কোন কিছু শুরু করতে বেগ পেতাম । & \\
\hline ১৭. আমার মনে হতো আমার আর কিছুই করার নেই । & \\
\hline ১৮. আমি মন খারাপ করে থাকতাম । & \\
\hline ১৯. আমি কোন কিছুতে উদ্দীপনা পেতাম না। & \\
\hline ২০. আমার নিজেকে মূল্যহীন মনে হত। & \\
\hline ২১. আমার জীবনকে অর্থহীন মনে হতো । & \\
\hline (0) আমার জন্য প্রযোজ্য নয় & \\
\hline (1) আমার জন্য কখনো প্রযোজ্য, কখনো নয় & \\
\hline (2) আমার জন্য বেশির ভাগ ক্ষেত্রেই প্রযোজ্য & \\
\hline (3) আমার জন্য প্রায়ই সবসময় প্রযোজ্য & \\
\hline
\end{tabular}

